\title{
Editorisches Interview: Demokratie in Zeiten des Internets
}

\author{
Peter Pagel ${ }^{1}$ Edy Portmann ${ }^{1}$ Joël Vogt ${ }^{1}$
}

๑) Springer-Verlag GmbH Deutschland, ein Teil von Springer Nature 2020

Das Internet prägt heute unser aller Leben, im Privaten wie im Beruflichen. Chefredakteur Peter Pagel sprach mit den Gastherausgebern dieses Schwerpunkts, Edy Portmann und Joël Vogt.

Peter Pagel: Welche Auswirkungen hat das Internet auf Demokratien?

Edy Portmann: Diese Frage adressiert unsere englischsprachige Mitherausgeberin Patricia Hetter Kelso, als US-Politikwissenschaftlerin und Witwe des berühmten US-Nationalökonomen Louis Kelso vom Kelso Institut, aus welchem viele der Texte in dieser Ausgabe des Informatik Spektrums ihre Kraft ziehen, in ihrem Essay auf sehr überzeugende Art und Weise. Auf Louis Kelso, den Pionier des Employee Stock Ownership Plans (ESOP), der es arbeitenden Menschen ermöglicht, Aktien ihrer Firma zu kaufen und diese aus zukünftigen Dividendenrendite zu bezahlen, geht auch der zweite Mitherausgeber, Joël Vogt, einem großen Kenner des Internets der Dinge sowie Experte für Beteiligungsformen im Internet, in seinem Artikel ein.

Joël Vogt: Ich habe diese Frage oft mit Patricia Kelso diskutiert. Dabei hat sie auch die Frage in den Raum gestellt, ob „Internet“ und „Demokratie“ überhaupt vereinbar sind. Ich denke schon, aber nicht in der aktuellen Form. Wir dürfen nicht vergessen, dass das Internet ein US-Militär Projekt zur Informationssammlung ist - Verteilung und Verwaltung. Und es wird heute mehr als je dazu verwendet; und nicht nur von Nachrichtendiensten, wo es immerhin oft Checks and Balances gibt, sondern auch von Privatunternehmen wie zum Beispiel Facebook, wo jedoch nicht nach nationaler Sicherheit optimiert wird, sondern nach Profit und Marktdominanz. Wir haben lange den Wert unserer persönlichen Daten nicht verstanden und vielleicht auch nicht realisiert, dass die digitale und physische Welt immer mehr

Peter Pagel

peter.pagel@ springer.com

1 Springer Fachmedien Wiesbaden $\mathrm{GmbH}$, Wiesbaden, Deutschland zusammenwachsen. Daten und Information sind Macht, sie ermöglichen oder zerstören ein demokratisches System.

Es ist kaum zehn Jahre her, als sich das Internet als Plattform für unsere Demokratie bewiesen hat. Die flexible und robuste Architektur unseres Internets, sowie die Benutzerfreundlichkeit des Webs (inkl. Tools und Protokolle) erlaubte etwa Aktivisten im Nahen Osten, sich zu organisieren und so Diktaturen zu stürzen. Ein paar Jahre später stellen wir aber mit Schrecken fest, dass ein Laissez-faire System auch eine grosse Bedrohung für die Demokratie und Menschenrechte sein kann: Brexit in Großbritannien, Trump in USA, der Genozid in Myanmar (Burma) wäre ohne das Internet, oder ohne gewisse Plattformen, nicht passiert. Wir dürfen nicht vergessen, dass unser Internetarchitektur zwar dezentral ist, aber Dienste sich, dank dem Netzwerkeffekt, sehr schnell auf wenigen Plattformen konzentrieren. Das heisst, Plattformen haben reellen Nutzen. Das Problem ist aber, dass anders als in einer Demokratie, Plattformen keine Gewaltentrennung kennen. Und wie zum Beispiel Mark Zuckerberg immer wieder zeigt, keine Rechenschaft ablegen müssen. Und obwohl grosse Plattformen oft $\mathrm{zu}$ öffentlichen Vorsorgeunternehmen wurden, haben wir Nichts davon.

Louis und Patricia Kelso haben genau zu diesem Zweck demokratisch-kapitalistische Finanzierungsmethoden entwickelt, welche dies ermöglichen. Wir dürfen aber auf keinen Fall das Internet überregulieren - dann ist es kein Internet mehr - aber auch auf keinen Fall es sich selbst überlassen. Denn die freie Marktwirtschaft blüht nur, wenn faire Rahmen- und Wettbewerbsbedingungen herrschen. Dazu braucht es aber klare und verständliche Regeln. Und da der Wert des Internets sowohl von Entrepreneuren und Steuergelder wie auch von Benutzern generiert werden, müssten Nutzer auch für ihren Beitrag entlohnt werden. Daten sind digitales Gold sowie der Rohstoff für maschinelles Lernen und künstliche Intelligenz. Wir haben also alle ein Interesse daran, dass diese Quelle sprudelt. Dienste werden dank KI besser, Wähler können sich informieren, Kunden werden für die Verwendung ihrer Daten entlohnt, Innovatoren und Forscher können ihre Ideen als Startups verwirklichen - und so weiter. Ich bin optimistisch, dass 
wir das Internet so ausbalancieren können, dass alle davon profitieren. Und ich muss optimistisch sein, denn die Alternativen machen mir Angst.

Edy Portmann: So geht es mir auch. Heutige System können (noch) tendenziös sein, was unterschiedliche Ursachen haben kann: Künstliche Intelligenz und ihr zugehöriges maschinelles Lernen benötigen eben Trainingsdaten, um Vorhersagen machen zu können. Aus dem Internet gesammelte Daten können aber befangen, verzerrt, unvollständig sowie ungenau - also unscharf - sein. Zum Beispiel klassifizierte vor ein paar Jahren ein Google-Algorithmus schwarze Menschen als Gorillas. Trotz grossen Anstrengungen war Googles bedrückende Lösung schlussendlich, den Bilderkennungsalgorithmus daran zu hindern, Gorillas zu erkennen.

Peter Pagel: Sharing Economy ist eines der gängigen Schlagworte, die mit dem Internet verknüpft sind, wie unterscheidet sich die Fiktion von der Wirklichkeit?

Edy Portmann: Das Konzept der Sharing Economy ist bestechend einfach: Alles, was nicht permanent genutzt wird, kann von uns Benutzern vermietet werden. Über eine Online-Plattform wird der Kontakt zwischen Anbieter und Nachfrager hergestellt. Die dem Konzept potentiell inhärente künstliche Intelligenz wird dabei immer stärker als Allzweckmittel und -technologie begriffen. Ähnlich der Elektrizität kann ihre Auswirkungen über alle Aspekte unseres Lebens verfolgt werden: von Use Cases im Gesundheitswesen, in der Industrie, im Finanzwesen und vielen mehr. Deshalb forscht beispielsweise Uber daran, wie sie ihre Daten nutzen könnten, um ihre Fahrer auch zu weniger rentablen Fahrten zu bewegen, um uns damit ein besseres Benutzererlebnis zu bieten.

Joël Vogt: Zurück zu Ihrer Frage von Fiktion oder Vision: So wie ich das verstehe, ist die Sharing Economy noch in den Kinderschuhen. Airbnb oder Couchsurfing sind erfolgreiche Beispiele; Uber wurde noch unter dem Vorwand der Sharing Economy lanciert, ist aber mehr ein Taxi-Unternehmen. Ich bin von der Sharing Economy begeistert, aber wir müssen sicher sein, dass wir nicht einfach Subscription-Modelle aus der digitalen Welt in die physische Welt übertragen. Für mich geht es bei einer Sharing Economy um Bürger, die ihre Ressourcen teilen und dafür entlohnt werden wolle. Als Beispiel: wenn ich mein Fahrzeug einer Sharing Economy zur Verfügung stelle, werde ich als Bürger und Kapitaleigentümer ,empowered“. Wenn ich aber ein selbstfahrendes Fahrzeug von Uber zum Bahnhof verwende, ist dies keine richtige Sharing Economy, sondern eine sehr gefährliche Konzentration von Kapital in den Händen eines Großunternehmens. Kapitalismus funk- tioniert nur, wenn wir alle Kapital haben. Konzentration von Kapital ist für mich kein wirklicher Kapitalismus - so war die Situation vor Adam Smith.

Edy Portmann: Angesichts der angestrebten Datenmonopole fragte sich der Kolumnist Philip Stephens einmal, wie man den Kapitalismus vor den Kapitalisten schützen könnte. Seine Erkenntnis: Kapitalismus erfordert Legitimität, er kann sich langfristig nur entfalten, wenn er das Wohlergehen von uns Bürgern im Auge hat.

Tech-Utopisten, die etwa in der künstlichen Intelligenz eine Neuauflage der unsichtbare Hand Adam Smiths sehen, weisen häufig darauf hin, dass überhaupt erst aus der Digitalisierung eine Sharing Economy gewachsen sei - und das stimmt! Es ist jedoch sehr wichtig, die Dynamik dahinter zu verstehen, die der modernen Sharing Economy inhärent ist. Wir stellen dabei fest, dass das Internet eine immer wichtigere Rolle in der Informations- und Machtasymmetrie spielt, die zwischen den Organisationen, die diese Dienste anbieten, sowie den Nutzern, die sie befüllen, bestehen. In einer meiner letzten Kolumnen in der Luzerner Zeitung, für welche ich regelmäßig schreibe, beschäftigte ich mich mit der digitalen Metamorphose unserer öffentlichen Infrastruktur. Dabei fragte ich mich, wie es wohl wäre, wenn Airbnb- oder Uber-ähnliche Plattformen im Besitz der Netzwerkindustrien wie der Post oder Telekommunikation wären und diese zum Wohle von uns verwalten würde.

In diesem Schwerpunkt des Informatik Spektrums präsentiert der Medienwissenschaftler Nathan Schneider Lösungen, indem er Louis Kelsos ESOP-Gedanken mit dem Internet erweitert. Ihm gemäss suchen heutige Onlineplattformen und auch Startups nämlich nach intelligenten Antworten auf Eigentumsfragen. Sein Artikel legt Strategien dar, welche den Sharing-Plattformen wie Airbnb, Lyft oder Uber als mögliche Vertrauensmechanismen respektive Enabler einer umfassenden, demokratischen Verantwortung uns Benutzern gegenüber dienen können. Zu diesem Zweck stützt er sich auf gemachte Erfahrungen mit (C)SOP-Mitarbeiterbeteiligung sowie weitere, innovative Arten von Eigentumsstrukturen. Nutzerorientiertes Vertrauen im Internet kann eine Governance und Gewinnbeteiligung unter den Beteiligten ermöglichen.

Peter Pagel: Vertrauen ist ein wichtiger Aspekt bei allem, was Menschen im Internet tun, kann die Blockchain-Technik da hilfreich sein?

Edy Portmann: Als möglichen Vertrauensmeachanismus fürs Internet stellt Nathan Schneider eben Alternativen, wie (C)SOP vor. Eine weitere Möglichkeit eruiert er zudem auch in der Blockchain-Technologie. Toni Caradonna von der Swiss Blockchain Federation, die sich mit der Schaffung von Rechtssicherheit sowie guten Voraussetzungen für 
das Schweizer Blockchain-Ökosystem einsetzt, geht in seinem Artikel genau dieser Technologie und deren Einsatz für die Gesellschaft auf den Grund. Dazu beleuchtet er deren Ursprung und stellt sie in den Kontext von drei Digitalisierungswellen: In der ersten geht es um die Digitalisierung von Informationen, die mit dem Aufkommen des Internet verdeutlicht wird, in der zweiten, manifestiert in und durch Social Media, um deren Communities und, in der dritten um Werten, repräsentiert durch Blockchain-Technologie. Er zeigt auf, wie Blockchain als neue Internettechnologie die Eigenschaften der Digitalisierung der ersten Wellen, wie etwa die Zentralisierung, die durch technologieunabhängige Geschäftsmodelle entstand, überwinden kann.

Peter Pagel: Könnte man sich Online-Marktplätze vorstellen, die besser zu Demokratien passen, als das bei den heutigen der Fall ist?

Joël Vogt: Absolut! Ich würde behaupten, heutige OnlineMarktplätze kreieren zu schnell Machtverhältnisse, welche den Verkäufer und auch den Kunden schaden können. Und es ist, wegen dem Netzwerkeffekt, eine Illusion, dass man einfach einen Marktplatz wechseln kann. Amazon ist zum Beispiel Marktplatz, Katalog, Verkäufer, Marktforschungsinstitut und Aufsicht in einem. Es ist bekannt, dass Amazon mit anderen Verkäufern konkurrenziert. Das können sie, da sie einfach mögliche Konkurrenten überwachen können und diese dann unterbieten. Diese haben aber keine Alternativen, Amazon ist ein zu wichtiger Kanal im westlichen Teil des Internets. Klagen können sie nicht - bei wem auch? Amazon?

Da Online-Marktplätze ein unentbehrlicher Teil der Geschäftstätigkeiten von Verkäufern sind, frage ich mich in meinem Artikel, wieso diese Unternehmen nicht gleich Eigentümer werden sollten. Louis Kelso hat dazu die CSOP erfunden. Bemerkung: Bei ESOP werden Angestellte Teilhaber ihrer Firma, bei CSOP können Benutzer und Kunden Teilhaber eines wichtigen Lieferanten, oder in unserem Fall eben eines Online-Marktplatzes werden. Verkäufer müssen sowieso Mitgliedergebühren zahlen, in einer CSOP würden diese mit der Zeit zu Teilhaber-Wertpapiere führen. Ich würde aber nicht einzigen bei einer Plattform bleiben, sondern eine Föderation von verknüpften Online-Marktplätze bilden, damit beispielsweise Unternehmer in China auf ihre Art und Weise die Plattform gestalten, aber trotzdem Kunden in den USA beliefern können. Der Verkauf könnte dabei über die US-Plattform abgewickelt werden. Somit profitieren die Eigentümer der US-Plattform, also die amerikanischen Verkäufer.

Ich erhoffe mir durch solche Maßnahmen eine Wirkung gegen Protektionismus. Vielleicht fragen sie sich nun, wieso ich nicht zuerst an Konsumenten gedacht habe. Das hatte ich eigentlich, aber nachdem Facebook (zusammen mit
Cambridge Analytica) unsere Wahl gehackt hat und danach auch diese in den USA, stellte ich fest, dass selbst in London, wo ich wohne, viele den Wert von persönlichen Daten (noch) unterschätzen. Mir wurde mir klar, dass die Zeit für eine CSOP für Konsumenten noch nicht reif ist.

Edy Portmann: Meines Erachtens ist Joël Vogts Beitrag für uns eine praktikable Form, auf welche ich in einer anderen LZ-Kolumne aufmerksam machte, in der ich mich fragte, wie wir uns vor Überwachungskapitalisten aus dem Silicon Valley schützen könnten. Ihr Kapitalismus ruft laut USÖkonomin Shoshana Zuboff in uns das ungute Gefühl aus, mit welchem uns die Silicon Valley-Giganten als Nutzer ihrer Plattformen heute eben überwachen. Heutige KI-Systeme profitiert von der Digitalisierung unserer Lebenswelt - dem Internet der Dinge sei etwa Dank -, die uns dazu gebracht hat, den Überwachungsapparat überhaupt erst zu entwickeln und welcher sowohl aus dem privaten wie auch aus dem öffentlichen Sektor gespeist wird. Immer mehr Organisationen verlassen sich darauf, Informationen zu sammeln, um ein scheinbar besseres Erlebnis zu ermöglichen. Zudem haben auch Regierungen im Namen der nationalen Sicherheit angefangen, gegen unsere Freiheiten und auf unser Recht auf Privatsphäre vorzugehen, wie etwa Aussagen von Informanten wie Edward Snowden, einem US-amerikanischer Whistleblower und ehemaligen CIA-Mitarbeiter, belegen.

Peter Pagel: Brauchen wir einen Systemwechsel?

Joël Vogt: Ja, wenn wir weiterhin in einer Demokratie leben wollen, müssen wir endlich verstehen, was Louis Kelso als Binary-Economics bezeichnet. Wir schaffen Vermögen nicht nur durch unsere Arbeitsleistung, sondern auch - und dass immer stärker und schneller -, durch Kapital. Wenn wir unser Vermögen nur durch Arbeitsleistung (er)schaffen wollen, werden wir immer ärmer, denn produktives Kapital verbessert sich exponentiell. Eine gesunde Demokratie braucht aber Wählerinnen und Wähler, welche wirtschaftlich eine solide Basis haben. Unsere Daten sind, wie gesagt, der Rohstoff des 21. Jahrhunderts, das digitale Gold. Daten ermöglichen eben maschinelles Lernen und künstliche Intelligenz. Je mehr Daten, desto fähiger - und produktiver - wird künstliche Intelligenz. Wenn wir unsere Daten einfach gratis weggeben, vergeben wir auch ein Teil unserer Stimme in der Demokratie. Facebook hätte nicht Kundendaten für politische Zwecke missbrauchen können, wären „ihre Kunden“ auch Inhaber ihrer eigenen Daten. Aber wenn wir unsere Daten als Kapital verwalten und investieren können, geht es noch weiter als individuelle Wahlen. Wir können sicherstellen, dass Startups nicht von Datensätze ausgeschlossen werden und dadurch Innovation fördern. Oder wir können auch die Evolution des Internets mitge- 
stalten indem wir entscheiden, in welche Unternehmen oder Themen wir unsere Daten investieren wollen.

Edy Portmann: Louis Kelso soll einmal gesagt haben, dass „die Menschen sich weigern, ein Problem zu erkennen, bis sie eine Lösung zu diesem Problem sehen“. So ging es mir hinsichtlich der in diesem Schwerpunktheft dargelegten Themen auch. Deshalb lege ich zum Abschluss dieses Heftes zur Demokratien im Zeitalter des Internets, als Abrundung, meinen Reifungsprozess als Schlusspunkt dar. Als ich mich nämlich vor ungefähr einem Jahr mit meinen beiden Mitherausgebern unterhielt, wusste ich noch nicht, welche Prozesse dies in mir auslösen würde. Als Informatiker kümmerte ich mich nämlich bis anhin noch relativ wenig um Technologie-Regulation. Das hat sich unterdessen dahingehend verändert, als dass ich mich auf europäischem Level mit der Regulation von Daten und zugehörigem maschinellem Lernen beteilige. Dies beleuchte ich in meinem Schlusspunkt.

Peter Pagel: Herr Portmann, Herr Vogt, besten Dank für das Gespräch. Es bleibt also spannend.

\section{Anhang}

\section{Zum Titelbild}

Die Abbildung zeigt rund 12.000 Zell-Tracks, welche die Entwicklung eines Zebrafisch-Embryos in den ersten zwölf Stunden nach Befruchtung darstellen. Neben der Bildaufnahmetechnik (Lichtblattmikroskopie), welche hochauflösende Einblicke in biologische Prozesse ermöglicht, ist besonders interessant, dass die Ergebnisse mit aktuellen WebTechnologien für jedermann erkundbar sind. Dank unseres Visualisierungstools kann jeder interessierte Wissenschaftler die biologischen Resultate der Publikation selbst im Browser seines Computers oder Smartphones betrachten und wird dabei durch verschiedene Methoden der Visualisierung, bspw. Edge Bundling, unterstützt.

Multi-scale imaging and analysis identify pan-embryo cell dynamics of germlayer formation in zebrafish Gopi Shah, Konstantin Thierbach, Benjamin Schmid, Johannes Waschke, Anna Reade, Mario Hlawitschka, Ingo Roeder, Nico Scherf \& Jan Huisken.https://www.nature.com/ articles/s41467-019-13625-0

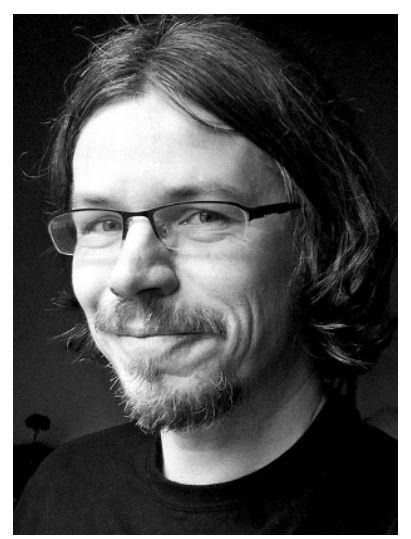

Peter Pagel

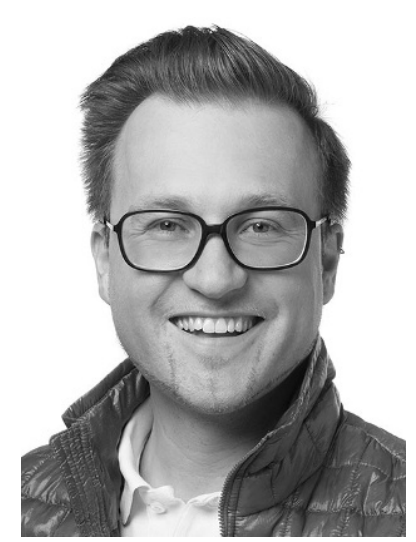

Edy Portmann

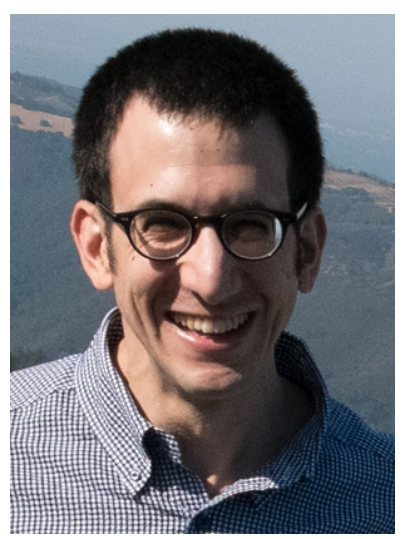

Joël Vogt 